\title{
Structural Insights into the Functional Domain of EmaA (Extracellular Matrix Protein Adhesin-A) from Aggregatibacter actinomycetemcomitans
}

\author{
Fereshteh Azari*, Michael Radermacher*, Keith Mintz** and Teresa Ruiz* \\ ** Dept. of Molecular Physiology and Biophysics, University of Vermont, Burlington, VT 05405 \\ * Dept. of Microbiology and Molecular Genetics, University of Vermont, Burlington, VT 05405
}

Adherence of the periodontal pathogen Aggregatibacter actinomycetemcomitans to host receptors is mediated by nonfimbrial adhesins $(1,2)$. The extracellular matrix protein adhesion-A (EmaA) is a $202 \mathrm{kDa}$ protein mediating the binding of this pathogen to collagen. EmaA belongs to Oca (Oligomeric coiled-coil adhesin) family of autotransporter adhesins and contains all of the conserved structural elements of the members of this adhesin family (3). Trimers of EmaA form flexible antenna-like appendages on the outer membrane surface of the bacterium. Our previous electron microscopy studies determined that the appendages could be described as long rods with at least 150 $\mathrm{nm}$ long. Some appendages are straight and others show bends at specific sites; the most prominent is localized at $29.4 \mathrm{~nm}$ from distal end and has a variable bend angle which ranges between $140^{\circ}$ $170^{\circ}$ from the bacterial surface $(3,4,5)$.

A combination of mutagenesis, collagen-binding activity measurements and electron microscopy determined that the functional domain comprised the $\mathrm{N}$ terminal domain of the EmaA molecule and was localized at the distal end of the appendages (4). Electron tomography and subvolume averaging of negatively stained whole mount bacteria, revealed the complex architecture of the functional domain which is composed of 3 subdomains (I, II and III) (5). Subdomain I has a globular shape with diameter of $5 \mathrm{~nm}$ and has been proposed to correspond to amino acids 70-206. Subdomains II and III have a cylindrical shape with dimensions $4.4 \mathrm{~nm}$ by $5.8 \mathrm{~nm}$ and $4.4 \mathrm{~nm}$ by $6.6 \mathrm{~nm}$, respectively and are separated by a linker region of $3 \mathrm{~nm}$ in length. Amino acids 70 to 386 were hypothesized to comprise subdomains I and II and the region of protein required for the formation of the functional collagen-binding domain (5). Electron tomography studies of three strains, wild-type strain and two mutant strains (in-frame deletions of amino acids 70-206 and 70-386) have been developed to examine this structural/functional hypothesis.

Single axis tomography tilt series were recorded from negatively stained whole mount bacteria for each of the strains and all the images in each tomographic series were aligned to a common origin using IMOD (6). 3D tomograms were reconstructed for each series using weighted backprojection algorithms in the SPIDER image processing system (7). Volume merging techniques were used to increase the signal-to-noise ratio of the reconstructions. EmaA subvolumes for each strain were windowed out of the tomograms, aligned and combined to obtain a single reconstruction containing a large number of projections. As more subvolumes of EmaA are aligned and merged together, the resolution of the structure increases and a more detailed 3D structure of the collagen-binding domain of EmaA with well-defined domains is generated. Our tomography data in the two mutant strains (deletion of amino acids 70-206 and 70-38) and the wild type strain have allowed us to interpret the features of each subdomain of EmaA in more detail and to correlate the 3D structure of the functional domain of EmaA with the amino acid sequence. 


\section{References}

1. D. H. Fine et al. (2005), Infect. Immun. 73:1947-1953.

2. K. P. Mintz. (2004), Microbiology 150: 2677-2688.

3. T. Ruiz et al. (2006), Infect. Immun. 74:6163-6170.

4. C. Yu et al. (2008), J. Bacteriol. 190: 3098-3109.

5. C. Yu et al. (2009), J. Bacteriol. 191: 6253-6261.

6. J. R. Kremeret et al. (1996), J. Struct. Biol. 116:71-76.

7. J. Frank et al. (1996), J. Struct. Biol. 116: 190-199.

8. This work was supported by NIH grant R01 DE017474 (awarded to T.R)



B



Figure 1: Electron micrographs of Aggregatibacter actinomycetemcomitans strains. A: Wild type strain (WT); B: emaA in $\Delta 70-206$ mutant strain; C: emaA in $\Delta 70-386$ mutant. Arrows indicate the head domain, including all subdomains in WT strain, Subdomain I in $\Delta 70-206$ mutant strain and subdomains I and II in $\Delta 70-386$ mutant strain. Scale bar: $100 \mathrm{~nm}$. 